\title{
Lesão do tendão flexor: sutura na região avascular ou vascularizada? Estudo biomecânico e histopatológico em coelhos*
}

\section{Flexor Tendon Injury: Avascular or Vascularized Region Suture? Biomechanical and Histopathological Study in Rabbits}

\author{
Trajano Sardenberg $^{1 \odot}$ Sergio Swain Muller ${ }^{1}$ Kunie labuk Rabello Coelho ${ }^{2}$ Denis Varanda ${ }^{1}$ \\ Andrea Christina Cortopassi ${ }^{1}$ Gilberto José Cação Pereira ${ }^{1}$ \\ Address for correspondence Trajano Sardenberg, MD, PhD, \\ Departamento de Cirurgia e Ortopedia, Faculdade de Medicina de \\ Botucatu, Universidade Estadual Paulista Júlio de Mesquita Filho \\ (UNESP), Av. Prof. Mário Rubens Montenegro s/n, Botucatu, SP \\ 18618687, Brasil (e-mail: tsarden@fmb.unesp.br).
}

\footnotetext{
${ }^{1}$ Departamento de Cirurgia e Ortopedia, Faculdade de Medicina de Botucatu, Universidade Estadual Paulista Júlio de Mesquita Filho (UNESP), Botucatu, SP, Brasil

2 Departamento de Patologia, Faculdade de Medicina de Botucatu, Universidade Estadual Paulista Júlio de Mesquita Filho (UNESP), Botucatu, SP, Brasil

Rev Bras Ortop 2019;54:268-274.
}

\section{Resumo \\ Palavras-chave \\ - traumatismos dos tendões \\ - coelhos \\ - suturas}

\section{Abstract}

Objetivos Analisar os aspectos mecânicos e histopatológicos da cicatrização do tendão flexor com interesse no local de colocação da sutura, na região vascularizada ou avascular. Métodos Um total de 83 coelhos foram submetidos à sutura central tipo Kessler na região de tendão vascularizado (grupo TN) e na de tendão avascular (grupo FC). $\mathrm{O}$ membro operado foi imobilizado por 3 semanas. Os animais foram sacrificados no período imediato, e a 2, 3 e 6 semanas de pós-operatório. As propriedades mecânicas estudadas foram: carga máxima, tensão na carga máxima, módulo de elasticidade, energia na carga máxima e energia por área. O tendão contralateral foi utilizado como controle. O estudo histopatológico foi descritivo.

Resultados A análise das propriedades mecânicas demonstrou comportamento semelhante em ambos os grupos, com estabilização ou discreto aumento no período imediato com 3 semanas e aumento acentuado com 6 semanas. A histopatologia demonstrou processo de cicatrização semelhante nos grupos TN e FC.

Conclusão A colocação da sutura central na região vascularizada ou fibrocartilaginosa avascular não apresenta diferenças em relação aos aspectos biomecânicos e histopatológicos na cicatrização do tendão flexor profundo dos dedos do pé do coelho.

Objectives To analyze the mechanical and histopathological aspects of flexor tendon healing with interest on the site of the placement of the suture, either in the vascular or in the avascular region.

\footnotetext{
* Trabalho realizado na Faculdade de Medicina de Botucatu Universidade Estadual Paulista Júlio de Mesquita Filho - UNESP. (D) Trajano Sardenberg's ORCID is https://orcid.org/0000-0001-61923149.
}

received January 29, 2018 accepted July 10, 2018
DOI https://doi.org/ 10.1055/s-0039-1692458. ISSN 0102-3616.
Copyright $(2019$ by Sociedade Brasileira License terms de Ortopedia e Traumatologia. Published by Thieme Revnter Publicações Ltda, Rio de Janeiro, Brazil 


\author{
Keywords \\ - tendon injuries \\ - rabbits \\ - sutures
}

\begin{abstract}
Methods A total of 83 rabbits were submitted to the Kessler type central suture in the vascularized tendon region (TN group) and in the avascular tendon region (FC group). The operated limb was immobilized for 3 weeks. The animals were sacrificed in the immediate postoperative period, and at 2, 3 and 6 weeks postoperatively. The mechanical properties studied were: maximum load, maximum load stress, modulus of elasticity, energy at maximum load, and energy per area. The contralateral tendon was used as control. The histopathological study was descriptive.

Results The analysis of the mechanical properties showed similar behavior in both groups, with stabilization or discrete increase in the immediate period at 3 weeks and a marked increase at 6 weeks. Histopathology demonstrated a similar healing process in the TN and FC groups.

Conclusion Placement of the central suture in the vascularized or avascular fibrocartilaginous region presents no differences in biomechanical and histopathological aspects in the healing of the flexor tendon in rabbits.
\end{abstract}

\section{Introdução}

No tratamento das lesões dos tendões flexores na zona II, a colocação da sutura central na região palmar do tendão, com a intenção de limitar a lesão vascular, é a orientação clássica., ${ }^{1,2}$ Porém, estudos experimentais concluíram que o posicionamento dorsal da sutura central apresenta maior resistência do que o palmar. ${ }^{3-7} \mathrm{~A}$ incerteza entre colocar a sutura central na região dorsal do tendão e obter maior resistência, eventualmente prejudicando a irrigação sanguínea do tendão ou colocar a sutura na região palmar para preservar a irrigação, porém com menor resistência, persiste na prática cirúrgica. ${ }^{8}$

O objetivo do presente estudo foi analisar os aspectos mecânicos e histopatológicos da cicatrização do tendão flexor com interesse no local de colocação da sutura, na região vascularizada ou avascular.

\section{Material e Métodos}

O projeto do presente estudo foi previamente aprovado pela Comissão de Ética em Experimentação Animal da instituição.

Foram utilizados 83 coelhos da raça Norfolk, do sexo masculino, com peso entre 1.500 g e 2.000 g e idade de $\sim 90$ dias.

Os animais foram divididos em grupos de acordo com a localização da sutura: grupo tendão normal (TN), com sutura

Tabela 1 Grupo Tendão Normal (TN): subgrupos, tempo de imobilização, tempo de seguimento pós-operatório, número de animais e atributos estudados

\begin{tabular}{|l|l|l|l|l|}
\hline Subgrupo & \multirow{2}{*}{$\begin{array}{l}\text { Imobilização } \\
\text { (semanas) }\end{array}$} & $\begin{array}{l}\text { Seguimento } \\
\text { (semanas) }\end{array}$ & & \multicolumn{2}{|l|}{ Número de animais $(\boldsymbol{n}=42)$} \\
\cline { 4 - 5 } & & Biomecânica & Histopatologia \\
\hline $\mathrm{TN}_{0}{ }^{*}$ & 0 & 0 & 6 & 0 \\
\hline $\mathrm{TN}_{2}$ & 2 & 2 & 6 & 4 \\
\hline $\mathrm{TN}_{3}$ & 3 & 3 & 6 & 6 \\
\hline $\mathrm{TN}_{6}$ & 3 & 6 & 6 & 8 \\
\hline
\end{tabular}

O número do subgrupo indica o tempo de seguimento em semanas. *Neste subgrupo, não foi realizada análise histopatológica. na região vascularizada; e grupo fibrocartilagem (FC), com sutura na região avascular. Cada grupo foi subdividido em quatro subgrupos de acordo com o tempo de seguimento pós-operatório, que foram de imediato e de 2, 3 e 6 semanas (-Tabelas 1 e 2 ).

O tendão utilizado foi o flexor profundo dos dedos do pé, na região do tornozelo, dentro de canal osteofibroso, envolto por tecido sinovial, onde apresenta nódulo de forma elíptica, no qual se observa uma região com epitendão e vasos sanguíneos, denominada na presente investigação de tendão normal, e uma região esbranquiçada e avascular, denominada de tendão fibrocartilaginoso. ${ }^{9-11}$ A lesão do tendão foi realizada por meio de acesso cirúrgico medial na região do tornozelo e secção transversal completa do tendão no centro do nódulo. O tendão contralateral foi utilizado como controle.

O reparo foi realizado com ponto tipo Kessler utilizando-se fio monofilamentar de náilon 4.0 (agulha triangular 2,0 cm, Point Suture, Fortaleza, Ceará, Brasil) colocado na região vascularizada no Grupo TN e na região avascular do tendão no Grupo FC, completada por sutura circunferencial com fio monofilamentar de náilon 6.0 (agulha cilíndrica $1,5 \mathrm{~cm}$, Ethicon, São Paulo, São Paulo, Brasil). A extremidade operada foi imobilizada com tala plástica mantendo o tornozelo e os dedos em extensão (-Fig. 1).

Tabela 2 Grupo Fibrocartilagem (FC): subgrupos, tempo de imobilização, tempo de seguimento pós-operatório, número de animais e atributos estudados

\begin{tabular}{|l|l|l|l|l|}
\hline Subgrupo & \multirow{2}{*}{$\begin{array}{l}\text { Imobilização } \\
\text { (semanas) }\end{array}$} & \multirow{2}{*}{$\begin{array}{l}\text { Seguimento } \\
\text { (semanas) }\end{array}$} & \multicolumn{2}{|l|}{ Número de animais $(\boldsymbol{n}=41)$} \\
\cline { 4 - 5 } & & Biomecânica & Histopatologia \\
\hline $\mathrm{FC}_{0}{ }^{*}$ & 0 & 0 & 6 & 0 \\
\hline $\mathrm{FC}_{2}$ & 2 & 2 & 6 & 5 \\
\hline $\mathrm{FC}_{3}$ & 3 & 3 & 6 & 5 \\
\hline $\mathrm{FC}_{6}$ & 3 & 6 & 6 & 7 \\
\hline
\end{tabular}

O número do subgrupo indica o tempo de seguimento em semanas. *Neste subgrupo, não foi realizada análise histopatológica. 

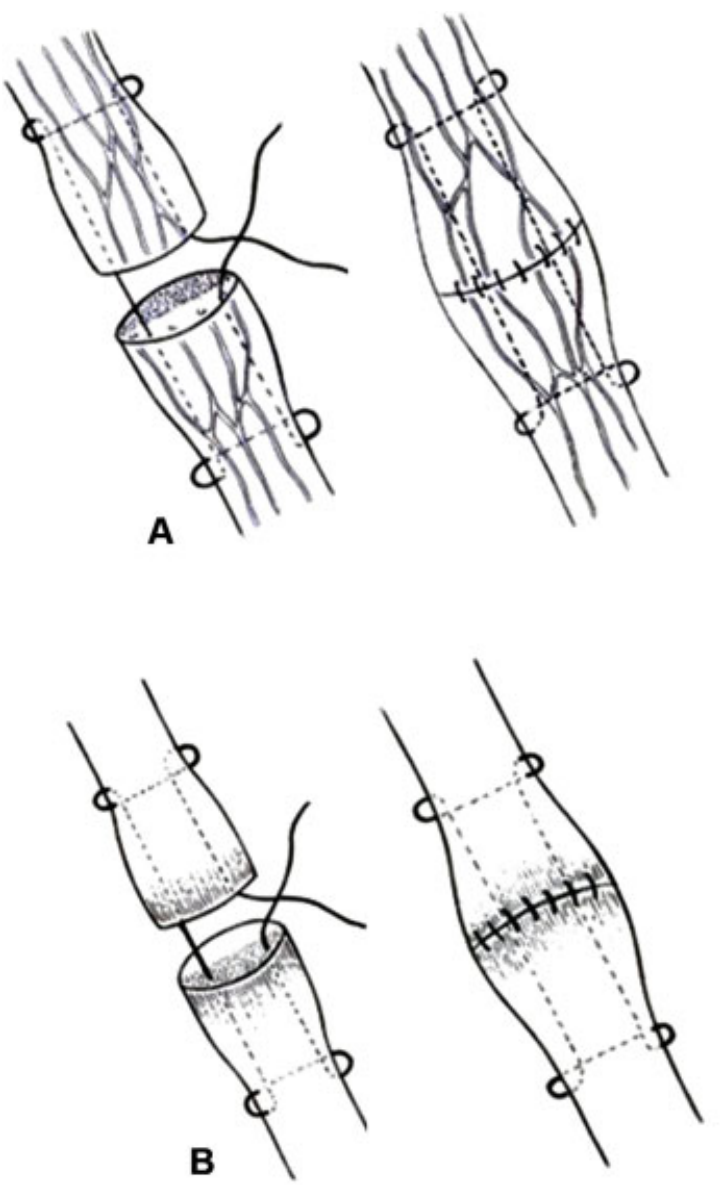

Fig. 1 (A) Sutura utilizada no grupo tendão normal vascularizado (TN). (B) Sutura utilizada no grupo fibrocartilagem avascular (FC). (C) Animal com o membro pélvico operado imobilizado.

Após o sacrifício dos animais, os tendões foram retirados no tamanho de $4 \mathrm{~cm}$, fixados em garras metálicas sinusoidais com distância de $20 \mathrm{~mm}$ e local da cicatrização central. As garras foram montadas axialmente em máquina universal de ensaio mecânico com célula de carga de 1000N (EMIC Equipamentos e Sistemas de Ensaio Ltda., Modelo DL 10000, Curitiba, Parana, Brasil) e velocidade de aplicação de carga de $30 \mathrm{~mm} /$ minuto. A área de secção do tendão foi obtida por meio da medida da área elíptica da região do nódulo do tendão contralateral não operado, sendo este valor utilizado no cálculo das propriedades mecânicas materiais dos tendões operados e não operados.

As propriedades mecânicas estudadas foram: carga máxima (N) e energia na carga máxima (N.10-3), denominadas de estruturais e, tensão na carga máxima (MPa), módulo de elasticidade (MPa) e energia por área (N.10-3/ $\mathrm{mm} 2$ ), denominadas de materiais, dependentes, portanto, da área de secção para o cálculo. Os aspectos histopatológicos foram estudados por meio de microscopia óptica com as colorações de hematoxilina e eosina (H\&E), tricrômico de Masson, e picrossíruis red com luz polarizada.

O estudo estatístico das variáveis das propriedades mecânicas, segundo o local da sutura central (grupos TN e FC), momentos experimentais (imediato, 2, 3 e 6 semanas) e lado (operado e controle contralateral) foi realizado pela técnica da análise de variância (ANOVA) não paramétrica para o

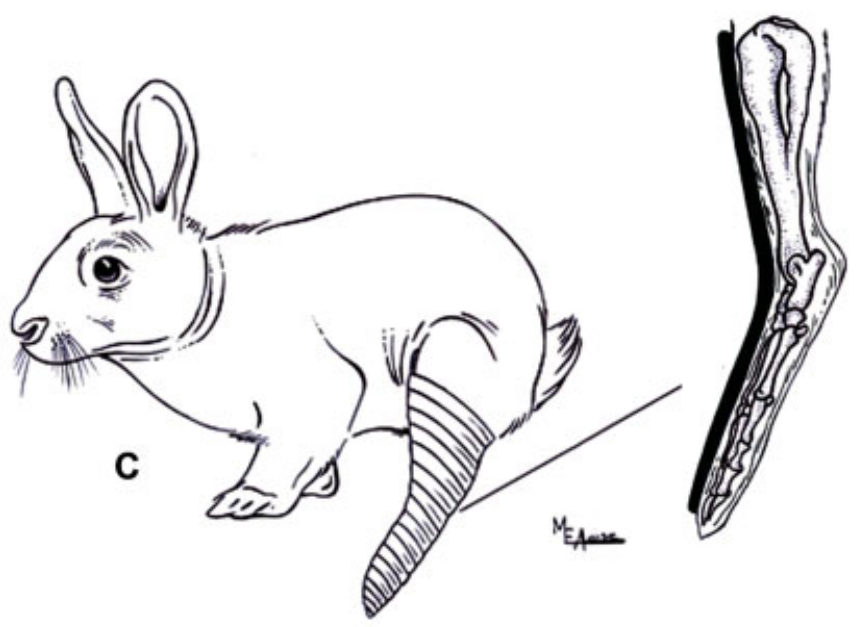

modelo de dois fatores em medidas repetidas, complementado com os respectivos testes de comparações múltiplas. ${ }^{12}$ A discussão dos resultados foi realizada no nível de $5 \%$ de significância.

\section{Resultados}

\section{Propriedades mecânicas}

As - Tabelas 3-7 apresentam as medianas, os valores mínimo e máximo das propriedades mecânicas estudadas em ambos os grupos (TN e FC), lado operado e controle, nos quatro momentos experimentais acompanhados pelas letras de comparação (análise estatística).

As - Figs. 2 e 3 ilustram os resultados obtidos para cada propriedade mecânica.

As rupturas ocorreram sempre no local da sutura ou da cicatrização dos tendões em ambos os grupos experimentais. Nos tendões íntegros não operados, utilizados como controle, as rupturas ocorreram sempre no segmento entre a garra e o nódulo.

\section{Análise Histopatológica}

O processo de cicatrização tendínea ocorreu de modo semelhante em ambos os grupos experimentais, havendo diferenças somente nos subgrupos, relacionadas à evolução temporal dos fenômenos observados. 
Tabela 3 Medidas descritivas (mediana, valor mínimo e máximo) da carga máxima $(\mathrm{N})$, segundo tempo de experimentação, tipo de sutura (tendão normal e fibrocartilagem) e lado (operado e controle)

\begin{tabular}{|l|l|l|l|}
\hline \multirow{2}{*}{$\begin{array}{l}\text { Tempo } \\
\text { (semanas) }\end{array}$} & Sutura & Lado \\
\cline { 3 - 4 } & & Controle & Operado \\
\hline \multirow{2}{*}{0} & TN & $189(138-254) \mathrm{aA} \beta$ & $25(22-29) \mathrm{aA \alpha}$ \\
\cline { 2 - 4 } & FC & $149(119-200) \mathrm{aA} \beta$ & $29(20-39) \mathrm{aA \alpha}$ \\
\hline \multirow{2}{*}{3} & TN & $172(149-228) \mathrm{aA} \beta$ & $29(19-33) \mathrm{aA \alpha}$ \\
\cline { 2 - 4 } & FC & $171(119-185) \mathrm{aA} \beta$ & $32(16-35) \mathrm{aA \alpha}$ \\
\hline \multirow{2}{*}{6} & TN & $195(119-240) \mathrm{aA} \beta$ & $29(15-40) \mathrm{aA \alpha}$ \\
\cline { 2 - 4 } & FC & $139(134-196) \mathrm{aA} \beta$ & $32(20-48) \mathrm{aA \alpha}$ \\
\hline & TN & $174(156-293) \mathrm{aA} \beta$ & $91(68-119) \mathrm{aB} \alpha$ \\
\cline { 2 - 4 } & FC & $208(185-300) \mathrm{aB} \beta$ & $89(55-98) \mathrm{aB} \alpha$ \\
\hline
\end{tabular}

Sutura: TN (tendão normal) / FC (fibrocartilagem); letras minúsculas: comparação de sutura, fixado momento; letras maiúsculas: comparação de tempo, fixados sutura e lado; letras gregas: comparação de lado, fixados tempo e sutura; letras iguais: semelhança; letras diferentes: diferença.

Tabela 4 Medidas descritivas (mediana, valor mínimo e máximo) da energia na carga máxima (N. $10^{-3} \mathrm{~m}$ ) segundo tempo de experimentação, tipo de sutura (tendão normal e fibrocartilagem) e lado (operado e controle)

\begin{tabular}{|l|l|l|l|}
\hline \multirow{2}{*}{$\begin{array}{l}\text { Tempo } \\
\text { semanas) }\end{array}$} & Sutura & Lado \\
\cline { 3 - 4 } & & Controle & Operado \\
\hline 0 & TN & $514(291-638) \mathrm{bA} \beta$ & $53(14-75) \mathrm{aA} \alpha$ \\
\cline { 2 - 4 } & FC & $282(209-522) \mathrm{aAB} \beta$ & $66(46-108) \mathrm{aAB} \alpha$ \\
\hline \multirow{2}{*}{2} & TN & $376(320-580) \mathrm{aA} \beta$ & $43(18-76) \mathrm{aA} \alpha$ \\
\cline { 2 - 4 } & FC & $383(216-463) \mathrm{aAB} \beta$ & $53(18-101) \mathrm{aA} \alpha$ \\
\hline \multirow{3}{*}{3} & TN & $374(184-524) \mathrm{aA} \beta$ & $34(12-54) \mathrm{aA} \alpha$ \\
\cline { 2 - 4 } & FC & $248(176-347) \mathrm{aA} \beta$ & $51(28-99) \mathrm{aA} \alpha$ \\
\hline \multirow{2}{*}{6} & TN & $412(308-899) \mathrm{aA} \beta$ & $139(87,5-239) \mathrm{aB} \alpha$ \\
\cline { 2 - 4 } & FC & $507(260-1043) \mathrm{aA} \beta$ & $107(78-169) \mathrm{aB} \alpha$ \\
\hline
\end{tabular}

Sutura: TN (tendão normal) / FC (fibrocartilagem); letras minúsculas: comparação de sutura, fixado momento; letras maiúsculas: comparação de tempo, fixados sutura e lado; letras gregas: comparação de lado, fixados tempo e sutura; letras iguais: semelhança; letras diferentes: diferença.

Com 2 semanas de evolução pós-operatória, quando o espaço entre os cotos tendíneos era amplo, ocorreu preenchimento principalmente por exsudato de fibrina e proliferação de tecido de granulação. Nas situações em que o espaço entre os cotos tendíneos era menor, houve preenchimento predominantemente por tecido de granulação com edema intersticial, focos irregulares de depósitos de fibrina e de discreto infiltrado inflamatório mononuclear permeado por poucos eosinófilos. Este tecido de granulação, na região central do espaço, proliferou a partir dos vasos do interstício dos cotos
Tabela 5 Medidas descritivas (mediana, valor mínimo e máximo) da tensão na carga máxima (MPa) segundo tempo de experimentação, tipo de sutura (tendão normal e fibrocartilagem) e lado (operado e controle)

\begin{tabular}{|l|l|l|l|}
\hline \multirow{2}{*}{$\begin{array}{l}\text { Tempo } \\
\text { (semanas) }\end{array}$} & Sutura & Lado \\
\cline { 3 - 4 } & & Controle & Operado \\
\hline \multirow{2}{*}{2} & TN & $22(16-32) \mathrm{aA} \beta$ & $3,1(2,7-3,4) \mathrm{aA} \alpha$ \\
\cline { 2 - 4 } & FC & $17(14-22) \mathrm{aA} \beta$ & $3,4(2,2-4,7) \mathrm{aA} \alpha$ \\
\hline \multirow{2}{*}{3} & TN & $21(18-27) \mathrm{aA} \beta$ & $3,5(2,2-3,8) \mathrm{aA} \alpha$ \\
\cline { 2 - 4 } & FC & $20(13-23) \mathrm{aA} \beta$ & $3,5(1,9-4,2) \mathrm{aA} \alpha$ \\
\hline \multirow{2}{*}{6} & TN & $22(15-27) \mathrm{aA} \beta$ & $3,7(1,9-4,3) \mathrm{aA} \alpha$ \\
\cline { 2 - 4 } & FC & $18(14-32) \mathrm{aA} \beta$ & $4,1(2,9-7,8) \mathrm{aAB} \alpha$ \\
\hline & TN & $21(18-40) \mathrm{aA} \beta$ & $11,9(7,5-13,6) \mathrm{aB} \alpha$ \\
\cline { 2 - 4 } & FC & $25(20-29) \mathrm{aA} \beta$ & $9,9(4,5-13) \mathrm{aB} \alpha$ \\
\hline
\end{tabular}

Sutura: TN (tendão normal) / FC (fibrocartilagem); letras minúsculas: comparação de sutura, fixado momento; letras maiúsculas: comparação de tempo, fixados sutura e lado; letras gregas: comparação de lado, fixados tempo e sutura; letras iguais: semelhança; letras diferentes: diferença.

Tabela 6 Medidas descritivas (mediana, valor mínimo e máximo) do módulo de elasticidade ( $\mathrm{MPa}$ ) segundo tempo de experimentação, tipo de sutura (tendão normal e fibrocartilagem) e lado (operado e controle)

\begin{tabular}{|l|l|l|l|}
\hline \multirow{2}{*}{$\begin{array}{l}\text { Tempo } \\
\text { (semanas) }\end{array}$} & Sutura & Lado \\
\cline { 3 - 4 } & & Controle & Operado \\
\hline \multirow{2}{*}{2} & TN & $119(95,5-193) \mathrm{aA} \beta$ & $16(15-49) \mathrm{aA} \alpha$ \\
\cline { 2 - 4 } & FC & $99(87-120) \mathrm{aA} \beta$ & $17(10-22) \mathrm{aA} \alpha$ \\
\hline \multirow{2}{*}{3} & TN & $114(89-131) \mathrm{aA} \beta$ & $31(2,8-41) \mathrm{aA} \alpha$ \\
\cline { 2 - 4 } & FC & $114(72-144) \mathrm{aA} \beta$ & $30(14-37) \mathrm{aA} \alpha$ \\
\hline \multirow{2}{*}{6} & TN & $164(121-219) \mathrm{aA} \beta$ & $35(12-43) \mathrm{aA} \alpha$ \\
\cline { 2 - 4 } & FC & $135(62-265) \mathrm{aA} \beta$ & $37(14-123) \mathrm{aA} \alpha$ \\
\hline & TN & $161(96-195) \mathrm{aA} \beta$ & $72(55-91) \mathrm{aB} \alpha$ \\
\cline { 2 - 4 } & FC & $119(96-261) \mathrm{aA} \beta$ & $71(40-148) \mathrm{aB} \alpha$ \\
\hline
\end{tabular}

Sutura: TN (tendã normal) / FC (fibrocartilagem); letras minúsculas: comparação de sutura, fixado momento; letras maiúsculas: comparação de tempo, fixados sutura e lado; letras gregas: comparação de lado, fixados tempo e sutura; letras iguais: semelhança; letras diferentes: diferença.

tendíneos com deposição irregular de fibras colágenas ainda imaturas. Em parte das amostras, a presença de pequeno espaço entre os cotos esteve acompanhada de deposição de fibras colágenas com disposição paralela ao eixo longitudinal do tendão, que se coraram pelo tricrômico de Masson e demonstraram fibras delicadas e refringentes quando examinadas na coloração de picrossírius red com luz polarizada. Nas zonas periféricas, o tecido de granulação se originou a partir dos tecidos peritendíneos, formando aderências com a derme e tecido sinovial circunjacentes; a deposição de colágeno 
Tabela 7 Medidas descritivas (mediana, valor mínimo e máximo) da energia/área (N.mm/ $\mathrm{mm}^{2}$ ) segundo tempo de experimentação, tipo de sutura (tendão normal e fibrocartilagem) e lado (operado e controle)

\begin{tabular}{|l|l|l|l|}
\hline \multirow{2}{*}{$\begin{array}{l}\text { Tempo } \\
\text { (semanas) }\end{array}$} & Sutura & Lado \\
\cline { 3 - 4 } & & Controle & Operado \\
\hline 0 & $\mathrm{TN}$ & $63(35-76) \mathrm{bA} \beta$ & $6,3(1,7-8,3) \mathrm{aA} \alpha$ \\
\cline { 2 - 4 } & $\mathrm{FC}$ & $33(25-60) \mathrm{aA} \beta$ & $7,7(5,0-12,8) \mathrm{aA} \alpha$ \\
\hline \multirow{2}{*}{2} & $\mathrm{TN}$ & $44(39-69) \mathrm{aA} \beta$ & $5,1(2-8,9) \mathrm{aA} \alpha$ \\
\cline { 2 - 4 } & $\mathrm{FC}$ & $44(26-55) \mathrm{aA} \beta$ & $6,3(2,1-11,0) \mathrm{aA} \alpha$ \\
\hline \multirow{3}{*}{3} & $\mathrm{TN}$ & $44(27-57) \mathrm{aA} \beta$ & $4,1(1,5-6,6) \mathrm{aA} \alpha$ \\
\cline { 2 - 4 } & FC & $38(21-55) \mathrm{aA} \beta$ & $7,1(4,2-15,8) \mathrm{bA} \alpha$ \\
\hline \multirow{2}{*}{6} & $\mathrm{TN}$ & $48(34-108) \mathrm{aA} \beta$ & $20(11-25) \mathrm{aB} \alpha$ \\
\cline { 2 - 4 } & FC & $56(39-87) \mathrm{aA} \beta$ & $13(7-18) \mathrm{aB} \alpha$ \\
\hline
\end{tabular}

Sutura: TN (tendão normal) / FC (fibrocartilagem); letras minúsculas: comparação de sutura, fixado momento; letras maiúsculas: comparação de tempo, fixados sutura e lado; letras gregas: comparação de lado, fixados tempo e sutura; letras iguais: semelhança; letras diferentes: diferença. nessas zonas apresentou disposição irregular. Em meio ao processo de reparação, identificaram-se granulomas do tipo corpo estranho que circundavam os fios de sutura e interrompiam a continuidade da deposição regular de colágeno.

Nos animais sacrificados com 3 semanas de pós-operatório, houve redução do edema intersticial no tecido de granulação, ausência dos depósitos de fibrina, presença de infiltrado inflamatório mononuclear mínimo e deposição de colágeno mais denso em relação ao observado no período de 2 semanas.

Na 6 ${ }^{a}$ semana de pós-operatório, a maior parte do tecido de granulação foi substituída por depósito de colágeno denso, de maneira semelhante ao tecido tendíneo dos cotos, em arranjo paralelo ao eixo longitudinal do tendão; quando corado pelo picrossírius red e examinado com luz polarizada, o colágeno reparativo foi caracterizado por fibras mais delicadas e refringência pouco menos intensa quando comparado ao observado no tecido tendíneo dos cotos. Os granulomas tipo corpo estranho que envolvem os fios de sutura e as aderências aos tecidos dérmicos e sinoviais nas zonas periféricas foram observados em todos os momentos experimentais, com progressivo aumento da densidade do colágeno cicatricial.
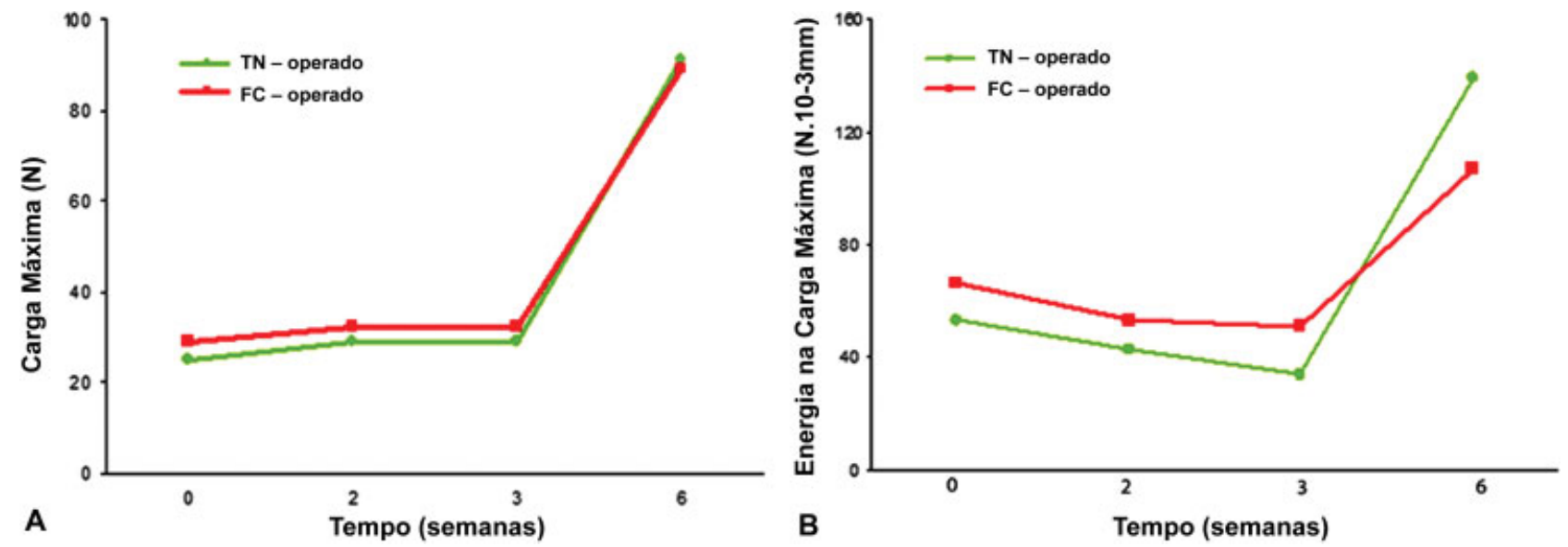

Fig. 2 Propriedades biomecânicas estruturais. (A) Medianas da Carga Máxima (N) dos grupos TN e FC, lado operado, nos quatro momentos experimentais; (B) Medianas da Energia na Carga Máxima $\left(\mathrm{N} .10^{-3} \mathrm{~m}\right)$ dos grupos TN e FC, lado operado, nos quatro momentos experimentais.
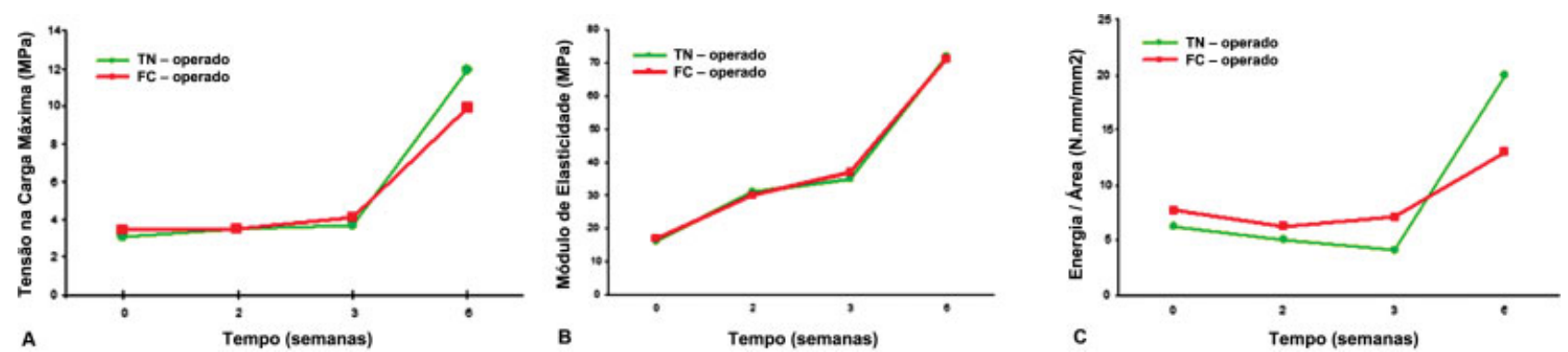

Fig. 3 Propriedades biomecânicas materiais. (A) Medianas da Tensão na Carga Máxima (MPa) dos grupos TN e FC, lado operado, nos quatro momentos experimentais; (B) Medianas do Módulo de Elasticidade (MPa) dos grupos TN e FC, lado operado, nos quatro momentos experimentais; (C) Medianas da Energia/Área (N.mm/mm2) dos grupos TN e FC, lado operado, nos quatro momentos experimentais. 


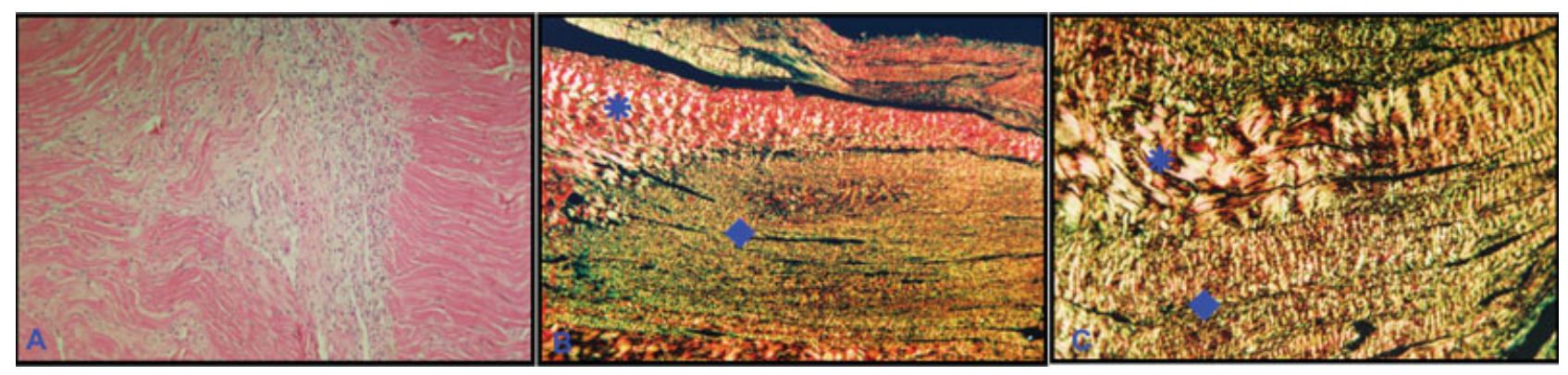

Fig. 4 (A) Duas semanas de pós-operatório - espaço entre os cotos encontra-se preenchido por tecido de granulação com discreto edema intersticial e infiltrado inflamatório mononuclear; observa-se, à esquerda do espaço, tecido de granulação a partir do interstício das fibras tendíneas do coto (Hematoxilina-eosina - aumento original 100x); (B) Três semanas de pós-operatório - presença de colágeno denso com intensa refringência no coto tendíneo $\left({ }^{*}\right)$, em continuidade com deposição de colágeno menos refringente e mais delicado, no espaço entre os cotos tendíneos() (picrossírius red com luz polarizada - aumento original 100x); (C) Seis semanas de pós-operatório - há distinção entre o colágeno do coto $\left({ }^{*}\right)$, mais denso e refringente, em relação ao colágeno cicatricial, mais delicado, menos refringente e com arranjo paralelo ao eixo longitudinal do tendão () (picrossírius red com luz polarizada - aumento original 100x).

A — Fig. 4 ilustra os resultados observados nos períodos de 2, 3 e 6 semanas de pós-operatório.

\section{Discussão}

O comportamento mecânico e morfológico dos tendões suturados foi semelhante na maioria dos parâmetros estudados nos animais dos grupos TN e FC, indicando que não houve diferenças na cicatrização tendínea em relação à região de localização do ponto central de sutura, na região de tendão normal vascularizada ou na região de tendão fibrocartilaginoso avascular.

Os ensaios mecânicos realizados imediatamente após o reparo (subgrupos $\mathrm{TN}_{0}$ e $\mathrm{FC}_{0}$ ) avaliaram a resistência inicial da sutura. Soejima et $\mathrm{al}^{4}$ e Stein et $\mathrm{al}^{13}$ realizaram estudos semelhantes por meio de ensaios mecânicos lineares e com tendões isolados. Soejima et al ${ }^{4}$ encontraram perfil mecânico superior na sutura colocada na face dorsal, enquanto Stein et $\mathrm{al}^{13}$ não observaram diferenças em relação ao local da sutura. Estudos semelhantes, porém por meio de ensaios mecânicos curvilíneos, demonstraram perfil mecânico superior na sutura colocada na face dorsal. ${ }^{3,5-7,14}$ É possível supor que o teste linear, realizado na presente investigação, anule 0 efeito mecânico de transmissão de maior tensão na face dorsal que na face palmar do tendão flexor. Além disso, a sutura periférica circunferencial contínua, que pode aumentar em até $50 \%$ a resistência do reparo central, pode ter equalizado os reparos realizados nas regiões de tendão normal e fibrocartilaginoso. ${ }^{15}$

Os ensaios mecânicos realizados nos períodos de 2, 3 e 6 semanas de pós-operatório avaliaram a resistência da cicatriz tendínea. As propriedades mecânicas estudadas nos grupos TN e FC não apresentaram diferenças estatísticas significativas, com exceção da energia por área no período de 3 semanas de pós-operatório, no qual o Grupo FC apresentou valores maiores do que o Grupo TN. Estes resultados indicam que não houve diferença no processo de cicatrização tendínea em relação à localização do ponto central da sutura. A colocação do ponto central da sutura na região vascularizada do tendão (Grupo $\mathrm{TN}$ ) poderia, em teoria, agredir o suprimento sanguíneo e prejudicar a cicatrização. No entanto, a imobilização da extremidade operada por 3 semanas no pós-operatório provavelmente favoreceu os mecanismos extrínsecos de cicatrização e diminuiu o possível efeito deletério da sutura na região vascularizada do tendão. A presença de aderências aos tecidos dérmicos e sinoviais, observadas na histologia em ambos os grupos e em todos os momentos experimentais, na região periférica da cicatriz tendinosa, sugere o predomínio dos mecanismos extrínsecos de cicatrização, fato também observado por Wada et al. ${ }^{16}$

No período de 3 semanas, a energia por área foi maior no Grupo FC. A energia por área pode ser caracterizada como a capacidade do corpo de prova de absorver impacto e, nos materiais viscoelásticos como tendões, está relacionada à quantidade de material e ao arranjo das fibras colágenas. É possível imaginar que, com 3 semanas, o Grupo FC tenha apresentado cicatriz com maior volume e arranjo de colágeno irregular, o que justificaria a maior capacidade de absorção de impacto. No entanto, tratou-se de achado pontual, uma vez que, com 6 semanas, os valores foram semelhantes em ambos os grupos.

A influência do tempo no processo de cicatrização tendínea foi semelhante em ambos os grupos experimentais, TN e FC, com estabilização dos valores das propriedades mecânicas do período imediato até 3 semanas de pós-operatório, seguido de aumento acentuado com 6 semanas. As exceções a este padrão ocorreram no grupo FC em relação à tensão na carga máxima, na qual se observou início da recuperação com 3 semanas, e em relação à energia por área, no mesmo grupo, com queda na $2^{a}$ semana e recuperação na $3^{a}$ semana. $\mathrm{Na}$ literatura consultada, não foram encontrados experimentos que variassem a colocação do ponto central da sutura na região vascularizada ou fibrocartilaginosa avascular do tendão e que avaliassem o processo de cicatrização. 0 estudo de Nessler et $\mathrm{a}^{17}$ relativo à cicatrização de regiões de tendão normal e de tendão fibrocartilaginoso avascular em tendões flexores de cães indicou desempenho morfológico e mecânico superiores da cicatrização da fibrocartilagem. No entanto, o modelo experimental foi de lesão parcial, sem material de sutura e movimentação ativa imediata no pósoperatório. Nessas circunstâncias, pode ter ocorrido 
predomínio de mecanismos intrínsecos de cicatrização tendinosa e a fibrocartilagem, com seu arranjo irregular do colágeno apresentou cicatriz mais precoce que o tendão normal, que exige maior tempo para o alinhamento das fibras de colágeno.

\section{Conclusões}

A análise dos resultados mecânicos e histopatológicos obtidos na presente investigação permitem concluir que a colocação do ponto central de sutura em região vascularizada do tendão não prejudicou o processo de cicatrização tendínea.

\section{Conflitos de Interesses}

Os autores declaram não haver conflitos de interesses.

\section{Referências}

1 Kleinert HE, Smith DJ Jr. Primary and secondary repairs of flexor and extensor tendon injuries. In: Jupiter JB, editor. Flynn's hand surgery. 4th ed. Baltimore: Williams \& Wilkins; 1991: 241-261

2 Culp RW, Taras JS. Primary care of flexor tendon injuries. In: Hunter JM, Mackim EJ, Callahan AD, editors. Rehabilitation of the hand: surgery and therapy. 4th ed. St. Louis: Mosby; 1995: 417-431

3 Aoki M, Manske PR, Pruitt DL, Kubota H, Larson BJ. Work of flexion after flexor tendon repair according to the placement of sutures. Clin Orthop Relat Res 1995;(320):205-210

4 Soejima O, Diao E, Lotz JC, Hariharan JS. Comparative mechanical analysis of dorsal versus palmar placement of core suture for flexor tendon repairs. J Hand Surg Am 1995;20(05):801-807

5 Komanduri M, Phillips CS, Mass DP. Tensile strength of flexor tendon repairs in a dynamic cadaver model. J Hand Surg Am 1996; 21(04):605-611
6 Stein T, Ali A, Hamman J, Mass DP. A randomized biomechanical study of zone II human flexor tendon repairs analyzed in a linear model. J Hand Surg Am 1998;23(06):1043-1045

7 Cao Y, Xie RG, Tang JB. Dorsal-enhanced sutures improve tension resistance of tendon repair. J Hand Surg [Br] 2002;27(02): 161-164

8 Beredjiklian PK. Biologic aspects of flexor tendon laceration and repair. J Bone Joint Surg Am 2003;85-A(03):539-550

9 Gillard GC, Reilly HC, Bell-Booth PG, Flint MH. The influence of mechanical forces on the glycosaminoglycan content of the rabbit flexor digitorum profundus tendon. Connect Tissue Res 1979;7 (01):37-46

10 Merrilees MJ, Flint MH. Ultrastructural study of tension and pressure zones in a rabbit flexor tendon. Am J Anat 1980;157 (01):87-106

11 Mills DK, Daniel JC. Development of functional specializations within the maturing rabbit flexor digitorum profundus tendon. Connect Tissue Res 1993;30(01):37-57

12 Normam GR, Streiner DL. Bioestatistics: the base essentials. St Louis: Mosby; 1994

13 Stein T, Ali A, Hamman J, Mass DP. A randomized biomechanical study of zone II human flexor tendon repairs analyzed in an in vitro model. J Hand Surg Am 1998;23(06):1046-1051

14 Xie RG, Zhang S, Tang JB, Chen F. Biomechanical studies of 3 different 6-strand flexor tendon repair techniques. J Hand Surg Am 2002;27(04):621-627

15 Strickland JW. Flexor tendons: acute injuries. In: Green DP Hotchkiss RN, Pederson WC, editors. Green's operative hand surgery. 4th ed. Philadelphia: Churchill Livingstone; 1999: 1851-1897

16 Wada A, Kubota H, Miyanishi K, Hatanaka H, Miura H, Iwamoto Y. Comparison of postoperative early active mobilization and immobilization in vivo utilising a four-strand flexor tendon repair. J Hand Surg [Br] 2001;26(04):301-306

17 Nessler JP, Amadio PC, Berglund LJ, An KN. Healing of canine tendon in zones subjected to different mechanical forces. J Hand Surg [Br] 1992;17(05):561-568 